Revue internationale P.M.E.

Économie et gestion de la petite et moyenne entreprise

\title{
Éditorial
}

\section{Jean Lorrain}

Volume 18, numéro 3-4, 2005

URI : https://id.erudit.org/iderudit/1008479ar

DOI : https://doi.org/10.7202/1008479ar

Aller au sommaire du numéro

Éditeur(s)

Presses de l’Université du Québec

ISSN

0776-5436 (imprimé)

1918-9699 (numérique)

Découvrir la revue

Citer ce document

Lorrain, J. (2005). Éditorial. Revue internationale P.M.E., 18(3-4), 7-10.

https://doi.org/10.7202/1008479ar

Ce document est protégé par la loi sur le droit d'auteur. L'utilisation des services d'Érudit (y compris la reproduction) est assujettie à sa politique d'utilisation que vous pouvez consulter en ligne.

https://apropos.erudit.org/fr/usagers/politique-dutilisation/
Cet article est diffusé et préservé par Érudit.

Érudit est un consortium interuniversitaire sans but lucratif composé de l’Université de Montréal, l'Université Laval et l'Université du Québec à Montréal. Il a pour mission la promotion et la valorisation de la recherche. https://www.erudit.org/fr/ 


\section{Éditorial}

Nous sommes particulièrement fiers de présenter ce numéro thématique de la Revue internationale PME consacré à l'entreprise familiale. L'objectif poursuivi par ce numéro est sans contredit de susciter chez les chercheurs pméistes de la communauté francophone un intérêt pour la problématique de l'entreprise familiale. Plusieurs raisons militent en faveur de la poursuite de cet objectif, mais attardons-nous sur les deux principales. La première raison est que dans la population des PME, autant dans les pays industrialisés que dans ceux en voie de développement, la très grande majorité des entreprises sont des entreprises familiales. Selon certaines estimations, ce serait le cas de plus de $70 \%$ des entreprises. L'importance du nombre de PME familiales justifie à elle seule que les chercheurs francophones portent une attention accrue à cette problématique de recherche. La deuxième raison est que ces chercheurs s'intéressent peu à ce champ d'étude. Un indicateur de l'intérêt des chercheurs pour un domaine de recherche est certes le nombre d'articles publié par ceux-ci dans les revues scientifiques. En nous limitant à une recension des écrits sur cette problématique dans la principale revue scientifique de langue française sur les PME, c'est-à-dire la Revue internationale PME, le nombre d'articles publiés à ce jour dans cette revue sur l'entreprise familiale est de quatre seulement. Exception faite de deux articles ayant figuré dans le premier numéro de la Revue en 1988, seulement deux articles ont été publiés depuis sur cet objet de recherche.

Ce quasi-désintéressement des chercheurs francophones pour les entreprises familiales s'explique difficilement en sachant que ce domaine de recherche connaît depuis quelques années chez les chercheurs anglo-saxons, en particulier les chercheurs états-uniens, une très grande popularité. Cet intérêt pour cette communauté scientifique se compare aisément à la popularité de l'entrepreneuriat et des PME dans les années 1980. Pour en savoir davantage sur l'engouement des chercheurs anglo-saxons pour ce domaine de recherche, nous avons réalisé une recherche documentaire sur la principale base de données informatisée en gestion, ABI/ inform (Proquest). Nous avons ainsi découvert que plus de 572 articles scientifiques sur l'entreprise familiale sont recensés par cette banque documentaire. Or, 355 articles, soit $62,1 \%$ de tous les articles recensés dans cette base de données, ont été publiés au cours des cinq dernières années. Nous observons également que de plus en plus de chercheurs anglo-saxons reconnus pour la qualité de leurs travaux dans le domaine de l'entrepreneuriat et des PME ont entrepris des recherches sur l'entreprise familiale; pour en nommer quelques-uns, mentionnons Barbara Bird, Robert Brockhau et Harold Welsch. Finalement, les deux principales revues américaines sur les PME et l'entrepreneuriat, Journal of Business Venturing et Entrepeneurship Theory and Practice, ont publié, depuis 2003, plusieurs numéros thématiques sur l'entreprise familiale. Ces observations font dire à certains que

(C) 2005 - Presses de l'Université du Québec

Édifice Le Delta I, 2875, boul. Laurier, bureau 450, Sainte-Foy, Québec G1V 2M2 • Tél.: (418) 657-4399 - www.puq.ca

Tiré de: Revue internationale P.M.E., vol. 18, nos 3-4, sous la direction de Louis Raymond • PME1803N

Tous droits de reproduction, de traduction et d'adaptation réservés 
l'entreprise familiale est un des champs de recherche dans les sciences de la gestion qui connaît en ce début de XXI siècle une forte croissance, en termes de travaux et de publications.

Malgré l'effort de plus de 50 ans de recherche et de réflexion sur l'entreprise familiale et l'émergence d'un corpus théorique intéressant, il reste que, selon les synthèses théoriques récentes, les besoins de connaissance dans ce domaine sont énormes. Les chercheurs en entreprise familiale se posent une multitude de questions. En d'autres termes, de vastes zones demeurent inexplorées dans ce champ de recherche. Les grandes questions auxquelles tentent de répondre les chercheurs en entreprise familiale par leurs travaux sont multiples et complexes. N'ayant pas l'ambition de recenser toutes les questions formulées par ceux-ci, il nous semble cependant utile de présenter les plus importantes.

La principale question que tous les chercheurs dans le domaine se posent et à laquelle plusieurs tentent de répondre est la suivante: comment peut-on définir l'entreprise familiale? Définir de façon théorique et opérationnelle le concept de l'entreprise familiale est indispensable pour délimiter les frontières de ce champ d'étude. De façon corollaire, les chercheurs s'interrogent sur les particularismes de l'entreprise familiale qui permettraient de la différencier de l'entreprise non familiale. Il est aisé de comprendre que, faute d'établir cette différenciation, en termes de gouvernance, de gestion et de dynamique organisationnelle, c'est la pertinence de la recherche dans ce domaine qui sera remise en cause. La problématique de la succession de l'entreprise familiale est à l'origine des recherches dans ce champ. Encore aujourd'hui, la succession demeure au cœur de beaucoup de travaux en entreprise familiale. Les chercheurs s'interrogent sur les facteurs de réussite de ce passage de la gouvernance et de la propriété de l'entreprise d'une génération à l'autre. En sachant que la très grande majorité des travaux dans ce domaine de recherche a été réalisée à ce jour auprès d'entreprises familiales états-uniennes, plusieurs s'interrogent sur la possibilité de généraliser les connaissances accumulées à ce jour en entreprise familiale à d'autres contextes socioculturels et économiques. Des chercheurs ont entrepris récemment des travaux pour tenter d'apporter une réponse à ce questionnement. En présentant ces quelques questions de recherche, notre intention est d'amener les chercheurs pméistes de la communauté francophone à prendre conscience des nombreuses opportunités de recherche qui s'offrent eux.

Les contributions qui suivent intéresseront le lecteur par la pertinence des questions de recherche. Les travaux rapportés dans ce numéro thématique cadrent parfaitement avec les grandes préoccupations relevées précédemment. D'abord, Jean-Christophe Boungou Bazika, de l'Université Marien-Ngouabi, présente une réflexion sur la définition et le rôle de l'entreprise familiale dans une perspective africaine. En se basant sur une analyse comparative Afrique/Occident des concepts de l'entreprise et de la famille, cet auteur redéfinit la notion d'entreprise familiale

Revue internationale P.M.E., vol. 18, $\mathrm{n}^{\text {os }} 3-4,2005$

(C) 2005 - Presses de l'Université du Québec

Édifice Le Delta I, 2875, boul. Laurier, bureau 450, Sainte-Foy, Québec G1V 2M2 - Tél.: (418) 657-4399 - www.puq.ca

Tiré de: Revue internationale P.M.E., vol. 18, nos $3-4$, sous la direction de Louis Raymond P PME1803N

Tous droits de reproduction, de traduction et d'adaptation réservés 
pour l'adapter à la réalité socioéconomique du continent africain. Ce faisant, il nous fait prendre conscience de la difficulté, voire de l'impossibilité, de formuler une définition universelle de l'entreprise familiale. Cette entité économique, selon les diverses particularités familiales, représente des réalités différentes d'un pays à un autre, d'un continent à un autre. L'entreprise familiale en Afrique, selon ce chercheur, remplit une multitude de fonctions qui la différencie de l'entreprise individuelle, par exemple une fonction technique d'incubateur ou une fonction de financement pour la création d'entreprise. Il décrit ces diverses fonctions. Avant de conclure, cet auteur se questionne sur l'avenir de l'entreprise familiale sur le continent africain, car plusieurs menaces planent sur son existence, entre autres l'urbanisation et l'individualisation.

Un des sujets traités dans plusieurs articles publiés dans ce numéro est celui de la succession dans la PME familiale. Une des préoccupations majeures des gens qui travaillent au développement économique durable est celle de la pérennité des entreprises familiales. Leur inquiétude repose sur le fait que dans les 10 prochaines années, selon certaines sources d'information, le nombre d'entreprises qui seront confrontées à cette réalité de la transmission de la direction et de la propriété augmentera, et ce, de façon très significative. Or, la succession entrepreneuriale dans les entreprises constitue un défi de taille. En effet, selon les résultats d'enquêtes réalisées sur ce sujet, cités par les contributeurs de ce numéro, les taux de réussite de cette transition sont très faibles.

Dans ce numéro thématique, plusieurs contributeurs abordent la problématique de la transmission d'entreprise. Le fruit de leur travail scientifique laisse entrevoir des pistes intéressantes de solution à ce problème de succession. En premier lieu, Louise Cadieux, de l'Université du Québec à Trois-Rivières, s'intéresse à la réussite de la succession de la PME familiale. Sachant que l'une des principales causes d'échec de la transmission de l'entreprise est l'incapacité du prédécesseur à se désengager psychologiquement de son entreprise, l'auteure s'interroge sur la façon dont les prédécesseurs ayant réussi la succession de leur entreprise se désengagent de celle-ci. Après avoir étudié cinq cas de succession d'entreprise, elle a développé un modèle qui décrit le processus de désengagement du prédécesseur. Le travail de cette chercheure met en évidence le long cheminement psychologique que poursuivent les prédécesseurs pour se détacher affectivement de leur entreprise et, ce faisant, devenir des ex-chefs heureux dans leur nouvelle vie de retraité des affaires.

Dans le même esprit, Louise St-Cyr et Francine Richer, de la Chaire de développement et de relève de la PME à l'École des HEC de Montéal, aborde l'épineuse question de l'effet de la planification successorale sur la réussite de la transmission des PME familiales. L'objectif que ces auteures poursuivent est de cerner les pratiques exemplaires qui garantissent le changement générationnel à la gouvernance de l'entreprise. En s'appuyant sur un modèle conceptuel de

Revue internationale P.M.E., vol. 18, nºs 3-4, 2005

(C) 2005 - Presses de l'Université du Québec

Édifice Le Delta I, 2875, boul. Laurier, bureau 450, Sainte-Foy, Québec G1V 2M2 • Tél.: (418) 657-4399 - www.puq.ca

Tiré de: Revue internationale P.M.E., vol. 18 , nos $3-4$, sous la direction de Louis Raymond $\cdot$ PME1803N

Tous droits de reproduction, de traduction et d'adaptation réservés 
planification successorale, dans lequel elles décrivent les principales activités à la base du processus de la transmission d'entreprise, ces chercheures, par leur étude auprès de 115 chefs de PME québécoises, mettent en évidence que les PME familiales ayant réussi le transfert générationnel de l'entreprise ont réalisé certaines activités de planification successorale, mais pas toutes. Ce faisant, en conclusion, elles s'interrogent sur les spécificités de la transmission d'entreprise en contexte de PME.

L'intégration du successeur dans l'entreprise familiale représente pour le prédécesseur tout un défi. L'acceptation par les employés, en particulier le personnel cadre, du successeur dans ses fonctions de principal dirigeant dans l'entreprise familiale est une condition sine qua non pour un transfert d'entreprise réussi. Dans la note de recherche, Vivi Koffi et Jean Lorrain, de l'Université du Québec à Trois-Rivières, se questionnent sur les stratégies comportementales que les entrepreneures adoptent pour faire accepter à leurs employés que leur enfant leur succède au poste de p.-d.g. Faute de recherche sur la question, en se basant sur la littérature en entrepreneuriat féminin, ces auteurs formulent l'hypothèse que les femmes entrepreneures, ayant un management d'entreprise différent de celui de leur homologue masculin, intégreront leur successeur d'une façon bien particulière, en usant de stratégies dites féminines. Dans leur texte, ces auteurs présentent un cadre conceptuel qui explique les différents comportements que les prédécesseures adoptent pour garantir l'intégration organisationnelle de leur progéniture.

Un article et deux notes de recherche hors thème complètent ce numéro. Sandrine Berger-Douce de l'IAE de Valenciennes amorce une réflexion sur le management environnemental en PME, axée sur une démarche collective permettant de dépasser les obstacles à l'engagement environnemental de ces entreprises. Au moyen d'une étude statistique et économétrique, Nazik Fadil, de l'École de management de Normandie, analyse l'impact de l'introduction en Bourse des moyennes entreprises françaises sur leur performance financière, et ce, à travers la conduite stratégique du décideur. Enfin, Pascal Philippart, de l'IAE de Lille, étudie la façon dont certains chercheurs français au statut de fonctionnaire utilisent le dispositif juridique novateur et complexe qui les autorise à créer une entreprise pour valoriser leurs travaux de recherche.

En présentant le fruit de ces travaux de recherche exemplaires, nous espérons que ce numéro thématique contribuera à susciter auprès de nos collègues un intérêt pour la recherche en entreprise familiale. Bonne lecture!

Jean Lorrain

Coordinateur de ce numéro spécial Université du Québec à Trois-Rivières

Revue internationale P.M.E., vol. 18, $\mathrm{n}^{\text {os }} 3-4,2005$

(C) 2005 - Presses de l'Université du Québec

Édifice Le Delta I, 2875, boul. Laurier, bureau 450, Sainte-Foy, Québec G1V 2M2 - Tél.: (418) 657-4399 - www.puq.ca

Tiré de: Revue internationale P.M.E., vol. 18, nos $3-4$, sous la direction de Louis Raymond - PME1803N

Tous droits de reproduction, de traduction et d'adaptation réservés 